\title{
MUSIC TUNE GENERATION BASED ON FACIAL EMOTION
}

\author{
Pooja Mishra \\ Professor, (SPPU)Dept. of Computer Engineering, \\ Dr. D. Y. Patil Institute of Engineering, \\ Management \& Research, Akurdi, Pune, India
}

\author{
Himanshu Talele \\ (SPPU)Dept. of Computer Engineering, \\ Dr. D. Y. Patil Institute of Engineering, \\ Management \& Research, Akurdi, Pune, \\ India
}

\section{Rohit Vidhate}

(SPPU)Dept. of Computer Engineering, Dr. D. Y. Patil Institute of Engineering, Management \& Research, Akurdi, Pune, India

\begin{abstract}
An individual's face is an important part of a human body and it especially plays an important role in knowing an individual's temperament. Educe the required input from the individual face can now be done directly employing a camera. This input will then be used in various ways. One of the applications of this input can be for extracting the information to analyze the temperament of an individual. This data can then be used to get a list of songs that comply with the "temperament" derived from the input provided earlier. This will result in removing the time consuming and tedious task of segregating or grouping songs into different lists manually and helps to bring into existence a good playlist based on an users' emotional features. Various algorithms have been developed and proposed for automating the playlist generation process. Facial Expression Based Music tune intents to scan and interpret the data and accordingly creating a playlist based the parameters provided. The scanning and interpreting include audio feature extraction and classification to get a list of songs belonging to a similar genre or to get a list of similar sounding songs. For mutual understanding and sharing feelings and intentions with each other human emotions are important. The emotions are manifested in verbal and facial expressions. This paper mainly focuses on what are the
\end{abstract}

Yogesh Sawarkar

(SPPU)Dept. of Computer Engineering, Dr. D. Y. Patil Institute of Engineering, Management \& Research, Akurdi, Pune, India

\section{Ganesh Naikare}

(SPPU)Dept. of Computer Engineering, Dr. D. Y. Patil Institute of Engineering, Management \& Research, Akurdi, Pune, India

methodologies available for detecting human emotions for developing emotion-based music tune. These methodologies will be then used to create a music player which will not only detect facial expression but will also generate unique music tune for user. Here we capture face and detect emotion using Viola Jones and CNN. The paper aims to tell how system will work and also playlist generation based on emotion classification. The application is so developed in such a way that it can manage content accessed by user, analyze the image properties and determine the temperament of the user which will be used to detect mp3 file properties so that they can be added into emotion-based play lists according to the temperament.

Keywords: Facial Emotion Detection, Facial Expression Recognition, Audio Feature Recognition, Emotion Based Music Tune Generation

\section{INTRODUCTION}

Music plays an important role in an individual's life. It is an important source of entertainment and is often associated with a therapeutic role. With the advent of technology and contiguous advancements in multimedia, sophisticated music players have been designed and have been enriched with numerous 
International Journal of Engineering Applied Sciences and Technology, 2020

Vol. 5, Issue 4, ISSN No. 2455-2143, Pages 616-621

Published Online August 2020 in IJEAST (http://www.ijeast.com)

features, including volume modulation, genre classification etc. Even though users' need is successfully addressed using this feature, user has to search his playlist for songs according to his emotions. In traditional music player, a user has to surf through his playlist on his own and select songs that would enlighten his mood and emotional experience. This method of choosing the songs is complex and time consuming and user would face a dilemma in finding appropriate song. The advent of Audio Emotion Recognition (AER) and Music Information Retrieval (MIR) equipped the traditional systems with a feature that automatically parsed the playlist, based on different classes of emotions.

In existing system user selects every time to play some tune for himself, so we introduce a system that detects user emotion to generate tunes. Users are bore when they listen same tune every time, so we create new tune for every time when user accesses the system. When user emotion is detected same, system generates same tune every time. This also becomes tiresome for the user to listen same song again and again. So, in our system we are using method that will generate new tune which will be advancement of previous generated tune. Hence whenever same facial expression is detected, new tune will be generated every time.

\section{LITERATURE SURVEY}

1. Paper Name: Facial Emotion Recognition for Human-Computer Interactions using hybrid feature extraction technique.

Author: Shoaib Kamal, Dr.Farrukh Sayeed, Mohammed Rafeeq

Description: Facial expression recognition is the most important criteria for effective Human Computer Interaction (HCI) as well as a medium to understand and communicate with children who cannot emote verbally. In this paper, we propose a feature extraction technique by embedding 2D-LDA and 2DPCA.The features extracted were then tested on standard classifiers i.e., Support Vector Machine (SVM) and K-Nearest Neighbourhood (KNN) classifiers. Facial expression images from JAFFE and Cohn-Kennedy databases were utilized for training as well as testing. Very high facial emotion recognition rate of $97.63 \%$ and $94.8 \%$ has been obtained with the proposed method for JAFFE and Cohn-Kanade databases respectively [1].

2. Paper Name: Face Detection And Tracking Using Viola-Jones Algorithm.

Author: Kowsalya.P, Sathya.K, Shubhashini.T,Vikram.S, Priyadharsni
Description: In this paper, the tracking of a person using unique code under live stream of a surveillance camera without using internet is proposed. This paper also includes GSM module which is used to send the location of the desired person as a text message to the authorized person. The accuracy of tracking the desired person is attained by using Viola-Jones algorithm [2].

3. Paper Name: Novel Approach to Face Expression Analysis in Determining Emotional Valence and Intensity with Benefit for Human Space Flight Studies.

Author: Adrian Dinculescu ; Cristian Vizitiu, Alexandru Nistorescu ; Mihaela Marin.

Description: This research presents a version of a simple algorithm for face expression analysis which targets to determine emotional valence and intensity with the help of less number of points, i.e eight Facial Characteristic Points and two types of textures in the face which results in better processing time. This is used as an alternative for statistical pattern recognition which is used to characterize the many software. Hence, it is concluded that the prospective implementation of herein algorithm for facial expression analysis would be an important add-on to the existent solutions. This solution can be used to perform evaluation, selection, training and recovery of individuals in complex professions, especially, of astronauts before, during and after prolonged human space flights [3].

4. Paper Name: Overview Of Face Recognition System Challenges.

Author: Ambika Ramchandra, Ravindra Kumar

Description: In this paper the challenges that are likely to occur while searching for an object match within the database are thoroughly discussed. For developing a computational model that recognizes and classifies objects is bit difficult. The stated that the main reason of difficulty is that different faces are complex and multidimensional and each of their expression has meaningful visual stimuli. There are many algorithms that can solve this problem. Hence, it is concluded that the magic of matching real-time objects with the database faces sometimes does not work even though many face recognition algorithms with high success rates are developed. Software used for Face recognition fails at times even though the suspect images were present in public and private database entries. Hence, to get a successful match of suspect, our database should have some process of training, because most of algorithms which are developed are non-parametric, which can be major drawback of the developed system [4]. 
5. Paper Name: An Accurate Algorithm for Generating a Music Playlist based on Facial Expressions

Author: Anukriti Dureha

Description: Manual segregation of a playlist and annotation of songs, in accordance with the current emotional state of a user, is labor intensive and time consuming. Various algorithms have been used to automate this process. However, the existing algorithms are slow, increase the overall cost of the system by using additional hardware (e.g. EEG systems and sensors) and have less accuracy. This paper presents an algorithm that automates the process of generating an audio playlist, based on the facial expressions of a user, for rendering salvage of time and labor, invested in performing the process manually. The algorithm proposed in this paper aspires to reduce the overall computational time and the cost of the designed system. It also aims at increasing the accuracy of the designed system. The facial expression recognition module of the proposed algorithm is validated by testing the system against user dependent and user independent dataset. After experiment it is found that, user dependent results give $100 \%$ accuracy. Although user independent results for joy and surprise are $100 \%$, for sad, anger and fear are $84.3 \%, 80 \%$ and is $66 \%$ respectively. For user independent dataset, $86 \%$ is the accuracy of emotion recognition algorithm. In audio, for joy and anger, recognition rates obtained are $95.4 \%$ and 90 $\%$ respectively, while it gives $100 \%$ accuracy for sad, sad-anger and joy-anger. $98 \%$ is the overall efficiency of the audio emotion recognition algorithm. Implementation and testing of the proposed algorithm are carried out using an inbuilt camera. Hence, the proposed algorithm reduces the overall cost of the system successfully. Also, on average, the proposed algorithm takes $1.10 \mathrm{sec}$ to generate a playlist based on facial expression. Thus, it yields better performance, in terms of computational time, as compared to the algorithms in the existing literature [5].

\section{EXISTING SYSTEM}

Music tune recommendation has been extensively studied from different angles, taking into account a variety of features and factors, that could influence the users' choices or preferences. The most common approaches are variations of collaborative filtering and content-based filtering. These methods perform satisfactory in the long run, but immediate preferences can be heavily influenced by a range of different factors and characteristics, which is generally referred to as 'context'.

\section{PROPOSED SYSTEM}

In our web application, user login to the system and user face is captured by laptop camera. After that, only face is detected by using Vialo Jones algorithm and it detects face accurately using Haar function. Then users face emotions are detected by using CNN. Then according to the users detected emotions system generates different new tunes and finally merges that tune and play merged tune for users, every time new tune is generated for the user if the same emotion is detected.

\section{PROPOSED SYSTEM ADVANTAGES}

- Generate new tunes of the user based on emotions.

- When user emotions are same then also generate new tunes.

- It is efficient and faster

\section{SYSTEM ARCHITECTURE}

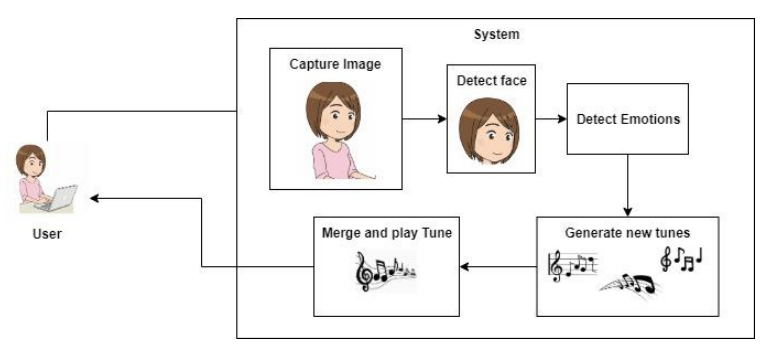

Figure 1 System Architecture

\section{ALGORITHM DETAILS}

- $\quad$ NN

- Step 1: Convolution Operation.

○ Step 1(b): ReLU Layer.

○ Step 2: Pooling.

- Step 3: Flattening.

- Step 4: Full Connection.

- Viola-Jones Algorithm

- Set the minimum window size, and sliding step corresponding to that size.

- For the chosen window size, slide the window vertically and horizontally with the same step.

- At each step, a set of $N$ face recognition filters is applied.

- If one filter gives a positive answer, the face is detected in the current widow. 
o If the size of the window is the maximum size stop the procedure.

O Otherwise increase the size of the window and corresponding sliding step to the next chosen size and go to the step 2 .

\section{IMPLEMENTED SYSTEM}

This web application helps the user to play music tunes based on his/her emotion detected using facial recognition. It will help user to enhance his mood. Important modules of web application are as below:

1. Main Screen (Welcome Screen):

When user opens web application he will get main screen which is welcome screen with three options to navigate between home page, registration page and login page.

\section{Registration/Login Page:}

Here, user will be able to register to website if he/she is a new user, else they can login on the website.

3. User Dashboard:

Once the user has been logged in successfully, he/she will get following options:

- View User Profile

- Capture Face

- Play Tunes

- Logout

4. Capture Face (Webcam):

This module will allow user to capture his face through webcam. Through the face captured using facial detection algorithm, facial emotion will be displayed and tunes will be generated based on emotion detected.

\section{Play Tunes Screen:}

User will be able to play tunes that has been generated based on his/her detected emotion.

\section{Recently Played Screen:}

In this module, user will be able to hover through different tunes that have been generated recently. User can play this tunes any number of times according to his or her wish.

7. Backend (MySQL Database):

In this database, registered user's information will be stored. Also, his/her detected emotions and generated tunes will be saved every time he/she captures their face and play the tune.
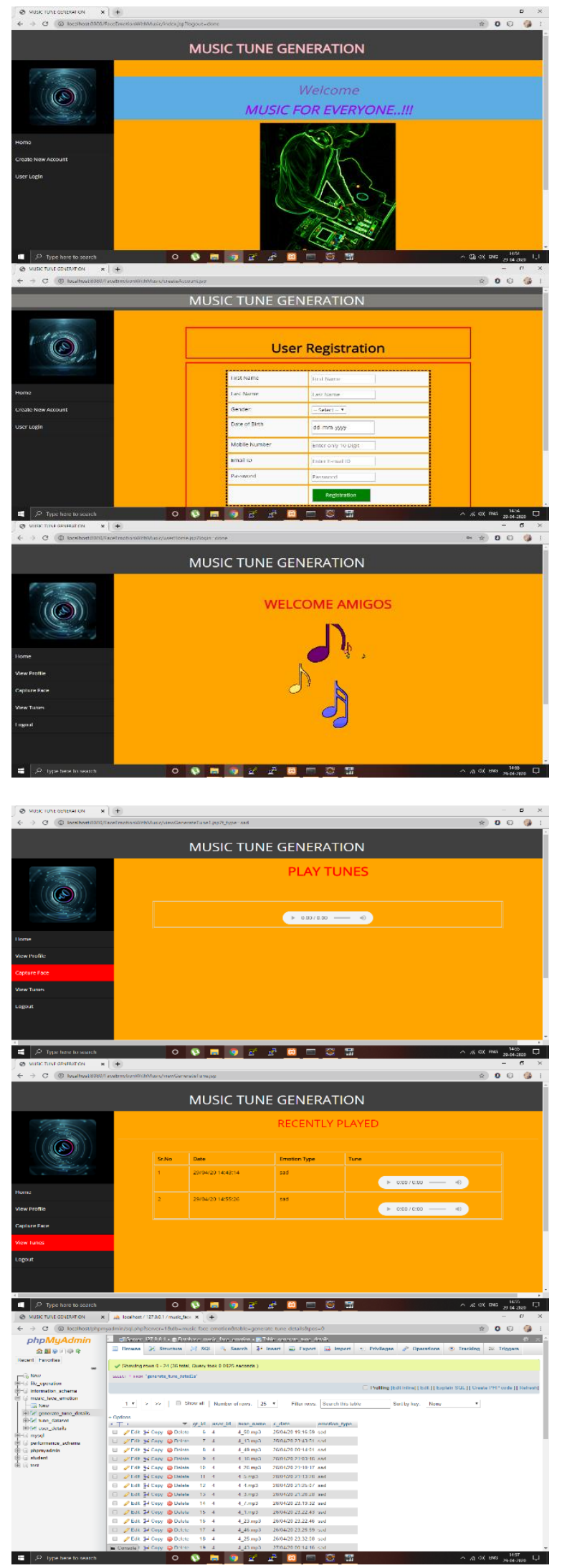
International Journal of Engineering Applied Sciences and Technology, 2020

Vol. 5, Issue 4, ISSN No. 2455-2143, Pages 616-621

Published Online August 2020 in IJEAST (http://www.ijeast.com)

\section{APPLICATION AND FUTURE SCOPE}

- Tutorial for beginner musicians

- Music Pattern Study

- Music Enhancement

- Music Testing

\section{CONCLUSION}

The algorithm proposed in this paper aims to generation music tunes based on facial expressions. Experimental results indicate that the proposed algorithm was successful in automating music tune generation on the basis of facial expressions and hence reduced the amount of labor and time, incurred in performing the task manually. The use of laptop or camera helped in eradicating the requirement of any additional hardware, such as EEG systems and sensors, and thus helped in curtailing the cost involved. Since, face emotion recognition is not performed in real time, the total time taken by the algorithm is equal to the amount of time taken by the algorithm to recognize facial expressions and the amount of time taken by the algorithm to query the meta data file. Hence, the proposed algorithm yields better performance, in terms of computational time, than the algorithms reported in the existing literature. Also, since the time taken by the algorithm to query the Meta data file is negligible $(0.0008 \mathrm{sec})$, the total time taken by the algorithm is proportional to the time taken to recognize facial expressions. Viola Jones algorithm Detect face of the user accurately. Then this gives input to the convolutional neural network and we get emotions.

\section{REFERENCES}

[1] Kamal Shoaib, Sayeed Dr.Farrukh, Rafeeq Mohammed (2011). "Facial Emotion Recognition for Human-Computer Interactions using hybrid feature extraction technique.", UKSim 5th European Simposium on Computer Modelling and Simulations 2011, 196-201.

[2] P Kowsalya, K Sathya, T Shubhashini, S Vikram, Priyadharsni (2018). "Face Detection And Tracking Using Viola-Jones Algorithm", Ijaerd Volume 5, Issue 04, April 2018.

[3] Vizitiu Cristian, Nistorescu Alexandru; Marin Mihaela, Dinculescu Adrian (2015). Novel Approach to Face Expression Analysis in Determining Emotional Valence and Intensity with Benefit for
Human Space Flight Studies", Published in E-Health and Bioengineering Conference, 19-21 Nov. 2015.

[4] Ramchandra Ambika, Kumar Ravindra (2013). "Overview Of Face Recognition System Challenges.", International Journal of Scientific \& Technology Research volume 2, issue 8, August 2013.

[5] Dureha Anukriti (2014). "An Accurate Algorithm for Generating a Music Playlist based on Facial Expressions", International Journal of Computer Applications (0975 - 8887) Volume 100- No.9, August 2014.

[6] Zhao Kun, Li Siqi, Cai Juanjuan, Wang Hui, Wang Jingling (2019). "An Emotional Symbolic Music Generation System based on LSTM Networks", 2019 IEEE 3rd Information Technology, Networking, Electronic and Automation Control Conference.

[7] Lopez-Rincon Omar, Starostenko Oleg, Ayala-San Gerardo Martín (2018). “Algoritmic Music Composition Based on Artificial Intelligence: A Survey", 978-1-5386-2363- 3/18/31.00 @2018 IEEE.

[8] Prof. Ghule Vijaykumar R., Benke Abhijeet B., Jadhav Shubham S., Joshi Swapnil (2017). "Emotion Based Music Player Using Facial Recognition", International Journal of Innovative Research in Computer and Communication Engineering, Vol. 5, Issue 2, February 2017 Copyright to IJIRCCE DOI: 10.15680/IJIRCCE.2017.

[9] Malgaonkar Saurabh, Biswajit Nag Yudhajit, Devadiga Rohit and Hirave Tejas (2012). "An AI Based Intelligent Music Composing Algorithm: Concord", Manuscript received September 8, 2012.

[10] Elliot W., Eck D., Roberts A., and Abolafia D (2016). "Project Magenta: Generating longterm structure in songs and stories,"2016,https://magneta.tensorflow.org/blog/20 16/07/15/lookback-rnn-attention-rnn/

[11] Boulanger-Lewandowski, N., Bengio Y., Vincent P. (2012). "Modeling temporal dependencies in high-dimensional sequences: application to polyphonic music generation and transcription," Proceedings of the 29th International Conference on Machine Learning, vol. 18, issue 13, pp. 3981-3991, 2012. 
[12] Hadjeres G., Pachet F., and Nielsen F. (2017). "Deepbach: a steerable model for bach chorales generation," Proceedings of the 34th International Conference on Machine Learning, pp. 1362-1371, 2017.

[13] Mogren O. (2016). "C-rnn-gan: continuous recurrent neural networks with adversarial training," Constructive Machine Learning Workshop (CML) at NIPS 2016 in Barcelona, S pain, 2016. 